\title{
Elaboration of Division-A Systematic Search for True Digits
}

\author{
Milosav M. Marjanović \\ Serbian Academy of Sciences and Arts, Belgrade, Serbia \\ Email: milomar@beotel.net
}

How to cite this paper: Marjanović, M.M (2017) Elaboration of Division-A Systematic Search for True Digits. Open Access Library Journal, 4: e3324.

https://doi.org/10.4236/oalib.1103324

Received: December 24, 2016

Accepted: February 18, 2017

Published: February 21, 2017

Copyright (C 2017 by author and Open Access Library Inc.

This work is licensed under the Creative Commons Attribution International License (CC BY 4.0).

http://creativecommons.org/licenses/by/4.0/

\begin{abstract}
In this paper, a new procedure of division is proposed and the corresponding didactical elaboration is sketched. A division task $A: B$ is called canonical when $A$ is less than tenfold $B$. As it is well known, each procedure of division splits into a number of canonical ones. The proposed elaboration is carried out in two main steps: the cases of canonical division by a two-digit divisor and the cases of long division. In order to make division easier, in the first step, a divisor is rounded up, increasing its first digit by 1 and replacing the second one by 0 . In the same time, the dividend is rounded down replacing its last digit by 0 . In this way the calculation is reduced to divisions by a one-digit divisor. This step is technically important and should precede the case of long divisions. Let $A: B$ be a case of canonical long division. The divisor $B$ is rounded up, increasing its second digit by 1 and replacing all those that follow by 0 's. In the same time, the dividend $A$ is rounded down, replacing by 0 's the same number of its final digits as in the case of $\mathrm{B}$. Thus, this division task is reduced to a "short" canonical division whose divisor is a two-digit number. According to a fact proved by this author in his paper Division-A Systematic Search for True Digits, II, The Teaching of Mathematics, XVIII, 2, (2015), 84-92, the quotient of the "short" division is equal or just 1 less than the number representing the true digit. This fact is the basis for the algorithm of producing true digits that we propose which is a contrast to the traditional "trial and error" method.
\end{abstract}

\section{Subject Areas}

Education

\section{Keywords}

Canonical Division, Long Division, Rounding up Divisor, Rounding down Dividend, Algorithm to Find True Digits 


\section{Introduction}

When writing this paper, we had the idea of entitling it "Learn to Divide", having in mind all possibly interested people and particularly, primary teachers and mathematics teachers. Namely, the procedures that are applied to sums, differences, products and quotients of two given natural numbers produce their value uniquely, except in the case of division when the "trial and error" method is applied. This method becomes more and more complicated with the increase of the component numbers (the dividend and the divisor), because it draws upon an improvised rounding of these numbers. Exercising this method of division, a dull routine is gained and it is an activity that the children dislike and the teachers usually leave this topic of arithmetic without a proper elaboration. Thanks to the pocket calculators, nowadays a skillful performance of arithmetic operations has lost its practical value, but their developmental significance stresses their elaboration as an important didactical task which will also occupy our attention in this paper.

Let $A: B$ represent a case of division where $A$ is the dividend and $B$ the divisor. When $A$ is less than tenfold $B$, such a case of division is called canonical. In canonical case the procedure of division consists of comparing $A$ with the multiples of $B: 0 \cdot B, 1 \cdot B, 2 \cdot B, \ldots, 9 \cdot B$ and $10 \cdot B$. When $k$ is a one-digit number such that $k \cdot B \leq A<(k+1) \cdot B$, then $k$ represents the true digit for $A: B$. As each procedure of division splits into canonical cases, we will be considering mostly such cases. A random comparison of $A$ with the multiples of $B$ leads to confusion which can be overcome by a systematic search for true digits.

Here, we intend to sketch a plan of gradual elaboration of division which is one of the main themes of arithmetic. At the top of this plan, we present an algorithm which directly produces the true digits and which is theoretically based on our papers [1] and [2]. Speaking less formally, our main result is the proof of the fact that, no matter how large a dividend and a divisor could be, the true digits are determined as a result of rounding up the divisor, increasing its second digit by 1 and replacing all of its digits that follow the second one by zeros and also rounding down the dividend replacing by zeros that the same number of its final digits. Though, all results in [1] and [2] are very elementary, this author considers them to be an essential contribution to the al-Khwarizmi's mathematics. We suggest to the reader to get acquainted with these results but it is not necessary for the following of the content of this paper.

Let us recall that Muhammad ibn Musa al-Khwarizmi (c. 780-c. 850) was a Persian mathematician whose original work on arithmetic, written in Arabic, was lost but its $12^{\text {th }}$ century Latin translation has been saved. This translation was untitled but its two first words Dixit Algorizmi (so said al-Khwarizmi) have been used for referring to it. This work of al-Khwarizmi was responsible for introducing the decimal positional number system to the Western Europe. Let us add also that the term algorithm is derived from the Latin form of the name al-Khwarizmi.

Beginning with the first grades of primary school the content of arithmetic is taught, first within the block of numbers up to 20 with the meaning of addition and subtraction being established and then, within the block of numbers up to 
100 with the meaning of multiplication and division also being established. In this stage of learning and teaching only simple calculation tasks are assigned to the children and everything else is subordinate to the establishing of the permanent meaning of the four arithmetic operations. "To establish meaning" is a phrase which is quite appropriate to the mode of learning at this level, when the children acquire the knowledge doing long series of exercises. These exercises have often the form of questions connected with various situations from the everyday life. The activity of doing such exercises results in the creation of mental images and schemes in the mind of a child. The permanent meaning of the arithmetic operations draws upon such mental representations whose creation is a subtle didactical task of arithmetic teaching.

In order to describe the schemes related to the arithmetic operations, the language of set theory has to be used, fixing in that way the type of exercises that are correspondent to each of the four arithmetic operations. In the case of multiplication and division, the corresponding scheme is a family of $m$ disjoint sets, each of which has $n$ elements and we call such a family of sets the multiplicative scheme. Let $p$ be the number of elements of the union of sets that belong to such a family. When $m$ and $n$ are given and $p$ is to be found, then we say that a multiplication task follows this scheme. When $p$ and $m$ (resp. $n$ ) are given and $n$ (resp. $m$ ) is to be found, then we say that a division task follows this scheme. In the first case, when $p$ and $m$ are given and $n$ is to be found, such a task is called partition and in the second case, when $p$ and $n$ are given and $m$ is to be found, such a task is called quotation.

The algorithms of performing arithmetic operations are based on already formed the fund which enters the long-term memory. That fund consists of addition table and that the same table when it is used for the purpose of subtraction-that is when the sum of two one-digit numbers is given together with the one of them and the table is used to see what is the other. Also multiplication table belongs to this fund as well as the division with the remainders: of numbers up to 19 by 2 , up to 29 by $3, \ldots$, up to 89 by 9 and up to 99 by 10 . Evidently, these are canonical divisions when divisors are one-digit numbers (and 10).

\section{Division When Divisors Are One-Digit Numbers or Powers of 10}

The procedure of division when divisors are one-digit numbers splits into a number of simple canonical divisions by $2,3, \ldots, 9$, as the following examples illustrate it:

(a)

$\begin{array}{lcc}\text { (i) } 87: 6=14 & \text { (ii) } 864: 5=172 & \text { (iii) } 5358: 7=765 \text {, etc. } \\ \underline{6} & \underline{5} & \underline{49} \\ 27 & 36 & 45 \\ \underline{24} & \underline{35} & \underline{42} \\ 3 & 14 & 38 \\ & \underline{10} & \underline{35} \\ & 4 & 3\end{array}$


Writing the equalities as, for example, the following are: $17234=1723 \times 10+$ $4,17234=172 \times 100+34,17234=17 \times 1000+234,17234=1 \times 10000+7234$, etc. in the form of division with remainders, we have

$$
\frac{17234}{4}: 10=1723, \quad \frac{17234}{34}: 100=172, \quad \frac{17234}{234}: 1000=17, \text { etc. }
$$

The equivalence of these two ways of writing is the explanation of this type of division.

These two cases of division cannot be extended to the general case of division by multi-digit divisors and therefore they are of less interest. Their elaboration needs, of course, some care but it is not among the aims of this paper.

\section{Canonical Division by Two-Digit Numbers}

Performance of this type of divisions is a very important technical skill and it has to run without any hesitation. To help calculation, a method of rounding will be described which is not applied in the following two cases:

(i) when dividend is less than divisor and when the quotient is 0 and

(ii) when dividend and divisor are two-digit numbers having the same first digits and when the quotient is 1 .

We exclude these trivial cases from our further considerations.

Let us consider now the case of division by two-digit divisors. The first step in performing such a division is rounding up the divisor, increasing its first digit by 1 and replacing its second digit by 0 and then, rounding down the dividend replacing its last digit by 0 . Division with the rounded components reduces to canonical division by one-digit divisor when the quotient is the first approximation of the true digit. If it is less than the number representing the true digit this procedure repeats and that new case of division is much easier, being the corresponding dividend smaller.

Before than we consider corresponding examples, let us note that we will abbreviate the propositions as, for example, they are: " 4 go into 27, 6 times", " 21 go into 31 , once" etc. simply writing: " 4 into $27: 6$ ", “21 into $31: 1$ ", etc. Calculations to be done aside (or mentally) are written in blue.

\begin{tabular}{|c|c|c|c|c|c|c|c|c|c|}
\hline \multicolumn{2}{|c|}{$271: 31=6$} & \multirow[t]{2}{*}{4 into $27: 6$} & \multirow{2}{*}{ (ii) } & \multicolumn{2}{|c|}{$85: 21=2}$, & \multirow[t]{2}{*}{3 into 8: 2} & \multicolumn{2}{|c|}{ (iii) $179: 22=5$, } & \multirow{2}{*}{3 into 17: 5} \\
\hline$\underline{186}$ & $\underline{2}$ & & & $\underline{42}$ & 1 & & $\underline{110}$ & 2 & \\
\hline 85 & 8 & 4 into $8: 2$ & & 43 & $\underline{1}$ & 3 into 4: 1 & 69 & $\underline{1}$ & 3 into $6: 2$ \\
\hline$\underline{62}$ & & & & $\underline{21}$ & 4 & & $\underline{44}$ & 8 & \\
\hline \multirow[t]{3}{*}{23} & & & & 22 & & 21 into $22: 1$ & 25 & & 22 into $25: 1$ \\
\hline & & & & $\underline{21}$ & & & $\underline{22}$ & & \\
\hline & & & & 1 & & & 3 & & \\
\hline
\end{tabular}

(b)

Instead of repeating divisions by one-digit numbers, that procedure can be shorten, performing mentally, in the second step, simple case of division by a two-digit number: 31 into 85: 2 (mental comparison: $2 \times 31=62<85<62+31$ =93), 21 into 43: 2 (m.c.: $2 \times 21=42<43<42+21=63$ ), 22 into 69: 3 (m.c.: 3 $\times 22=66<69<66+22=88)$. 
According to a result from [2], after the first step in the procedure of this division is done, a case of division remains where the dividend is less than 200 and when the first digit of the divisor is $9,8,7$ or 6 , the quotient is at most 2 , and when that digit is 5 or 4 , the quotient is at most 3 and finally when that digit is 3 or 2 , the quotient is at most 4 . Hence all divisions that remain in the second step are very easy for mental performing. Examples of such performing just follow:

(c)

\begin{tabular}{|c|c|c|c|c|c|c|c|c|}
\hline \multicolumn{2}{|c|}{ i) 179:22 = 5, } & \multirow[t]{2}{*}{3 into 17: 5} & \multicolumn{2}{|c|}{ (ii) $587: 94=5$, } & \multirow[t]{2}{*}{10 into $58: 5$} & \multicolumn{2}{|c|}{ (iii) $419: 52=6$, } & \multirow[t]{2}{*}{6 into $41: 6$} \\
\hline$\underline{110}$ & $\underline{3}$ & & $\underline{470}$ & $\underline{1}$ & & $\underline{312}$ & $\underline{2}$ & \\
\hline 69 & 8 & 22 into $69: 3$ & 117 & 6 & 94 into $117: 1$ & 107 & 8 & 52 into 107: 2 \\
\hline 66 & & & 94 & & & $\underline{104}$ & & \\
\hline 3 & & & 23 & & & 3 & & \\
\hline
\end{tabular}

To train this performance, first somewhat simpler cases of division, when both the dividend and the divisor are two-digit numbers, could be practiced. (Let us note also that it seems easier to multiply mentally a two digit number by a one-digit number, multiplying tens and units and then summing up these products. For example, $3 \times 27=3 \times 20+3 \times 7=60+21=81$, etc.). The related examples follow:

(d)

\begin{tabular}{|c|c|c|}
\hline (i) $81: 41=1$ & (ii) $96: 31=3$ & (iii) $87: 35=2$ \\
\hline$\underline{41}$ & $\underline{93}$ & $\underline{70}$ \\
\hline 40 & 3 & 17 \\
\hline$(1 \times 41<81<41+41=82)$ & $(3 \times 31=93<96<93+31=124)$ & $(2 \times 35=70<87<70+35=105)$ \\
\hline (iv) $91: 38=2$ & (v) $94: 28=3$ & (vi) $78: 22=3$ \\
\hline$\underline{76}$ & $\underline{84}$ & $\underline{66}$ \\
\hline 15 & 10 & 12 \\
\hline $2 \times 38=76<91<76+38=114)$ & $(3 \times 28=84<94<84+28=112)$ & $(3 \times 22=66<78<66+22=88)$ \\
\hline
\end{tabular}

When the last digit of divisor is 0 , there is no need to increase its first digit. In such cases the procedure runs as follows:

$712: 80=8, \quad 8$ into $71: 8, \quad 399: 60=6, \quad 6$ into $39: 6, \quad 378: 50=7, \quad 5$ into $37: 7$, etc. $\underline{640}$ $\underline{360}$ $\underline{350}$

72 39

28

When carried out mentally, "short" divisions require concentration of one's own attention on all related details. Then, they can be done without much effort and accurately. Everything is done as in the easier cases when two digit numbers are divided by a two-digit divisor. Let us take some examples: 43 into 243: 5 ( $5 \times$ $43=215<243<215+43=258), 79$ into $521: 6(6 \times 79=474<521<474+79=$ 553), 81 into $582: 7(7 \times 81=567<582<567+81=648)$, etc. This remark does not mean that we suggest that children would do these comparisons mentally. But when one is trained enough, this calculation goes quite easily.

\section{Division in Case of Multi-Digit Component Numbers}

For the beginning, let us consider the usual procedure of dividing, when both, dividend and divisor are multi-digit numbers (a long division). In this case, as it 
happens in general, the procedure of division splits into a number of canonical divisions, each of which produces a true digit.

(e)

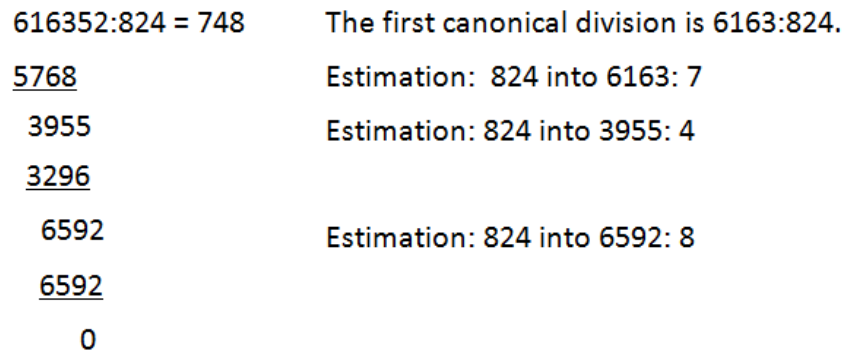

Apparently, this procedure runs smoothly as long as the way of estimation is not questioned. And instead of uncertain estimations, when the true digits are guessed, we propose an accurate procedure of producing true digits, which we will just present.

Let $A: B$ be a canonical division, where $A$ and $B$ are multi-digit numbers. Let $B$ be rounded up, increasing its second digit by 1 and replacing all those that follow by 0 's. Let $A$ be rounded down replacing by 0 's the same number of its final digits as it has been done in the case of $B$. Then, this division task reduces to a "short" canonical division whose divisor is a two-digit number. According to a fact proved in [2], the quotient of the "short" division is equal or just 1 less than the number representing the true digit. This fact is the theoretical basis for the algorithm of producing true digits that we propose here. Let us reconsider the example under (e), where all "short" divisions will be written aside.

$\left(\mathrm{e}^{\prime}\right)$

(i) $\begin{aligned} & 6163: 824=7 \\ & \underline{5768}\end{aligned}$

395

(ii) $3955: 824=4$

$\underline{3296}$

659

(iii) $6592: 824=7$

$\begin{array}{rr}\frac{5768}{824} & 1 \\ \frac{824}{0} & \end{array}$

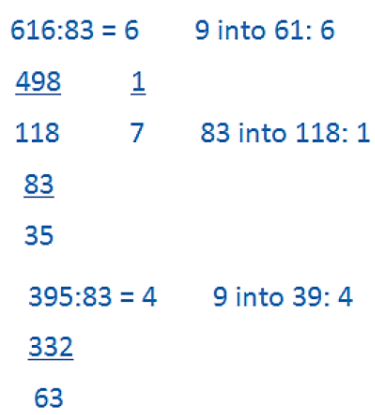

In the cases (e')(i) and (e')(ii), when dividing aside the true digit has been obtained and in the case (e')(iii) the trial number that has been obtained, is 1 less than the number represented by the corresponding true digit. Before proceeding further, we point out that in the following two cases, no rounding of component numbers is made. Let $A: B$ be a canonical division. Then,

(i) if $A<B$, the true digit is 0

and 
(ii) if $A \geq B$ and $A$ and $B$ have the same number of digits and their first digits are equal, the true digit is 1 .

Now examples of long division follow, where corresponding divisions by a two-digit number are supposed to be performed mentally (The mental calculation is intended for teachers but we suggest that children write aside and calculate in the written form).

(f)

(i) $84275: 32451=2,33$ into $84: 2$ (ii) $6534: 1283=5,13$ into $65: 5$ (iii) $81248: 13139=5$, 14 into $81: 5$ $\underline{64902}$

19373

(iv) $103011: 11993=8,12$ into $103: 8$ (v) 2894512:413291 = 6, 42 into 289: 6

$\begin{array}{rlll}\underline{95944} & \underline{2479746} & \underline{1} & \\ 7067 & & 7 & 413291 \text { into 424766: } 1 \\ & \underline{413291} & & \\ 1475 & & \end{array}$

When all final digits of a divisor, starting with the third one, are 0's, its second digit needs not be increased by 1 and the procedure of dividing runs as it is done in the following examples:

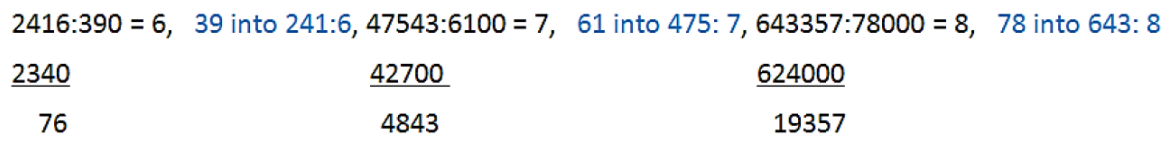

At the end let us present the procedure of dividing when the given division tasks are not canonical. Of course, nothing essentially new will be demonstrated as these divisions split into canonical ones.

(g)

\begin{tabular}{|c|c|c|c|c|}
\hline (i) 291812 & 770 & 38 into $291: 7$ & (ii) 1799383:4921 = 365, & 50 into $179: 3$ \\
\hline$\underline{2618}$ & 1 & & $\underline{14763}$ & \\
\hline 3001 & 780 & 38 into $300: 7$ & 32308 & 50 into 323: 6 \\
\hline$\underline{2618}$ & & & $\underline{29526}$ & \\
\hline 383 & & 374 into $383: 1$ & 27823 & 50 into $278: 5$ \\
\hline$\underline{374}$ & & & $\underline{24605}$ & \\
\hline 92 & & 374 into $92: 0$ & 3218 & \\
\hline$\underline{0}$ & & & & \\
\hline 92 & & & & \\
\hline (iii) & & & & \\
\hline 41328850 & $=82$ & 4, 51 into $413: 8$ & & \\
\hline 400816 & & & & \\
\hline 124725 & & 51 into $124: 2$ & & \\
\hline$\underline{100204}$ & & & & \\
\hline 245210 & & 51 into $245: 4$ & & \\
\hline$\underline{200408}$ & & & & \\
\hline & & & & \\
\hline
\end{tabular}


Everything is quite easy, isn't it?

We conclude with the remark that, as a part of the routine, some or all calculations done aside can also be carried out mentally. How to elaborate this theme with all details that contribute to a more routine performance of division should depend on a particular teacher and his/her class. Our aim was to sketch how to plan its gradual elaboration.

We hope that this paper will be a motivation for teachers to elaborate the theme of division with all necessary details that their class requires.

\section{References}

[1] Marjanovic, M.M. (2005) Division-A Systematic Search for True Digits. The Teaching of Mathematics, VIII, 89-101.

[2] Marjanovic, M.M. (2015) Division-A Systematic Search for True Digits, II. The Teaching of Mathematics, XVIII, 84-92. http://www.elib.mi.sanu.ac.rs/journals/tm

Submit or recommend next manuscript to OALib Journal and we will provide best service for you:

- Publication frequency: Monthly

- 9 subject areas of science, technology and medicine

- Fair and rigorous peer-review system

- Fast publication process

- Article promotion in various social networking sites (LinkedIn, Facebook, Twitter, etc.)

- Maximum dissemination of your research work

Submit Your Paper Online: Click Here to Submit

Or Contact service@oalib.com 